\title{
Molecular features of 23 patients with glycogen storage disease type III in Turkey: a novel mutation p.R1147G associated with isolated glucosidase deficiency, along with 9 AGL mutations
}

\author{
Yoshiko Aoyama ${ }^{1}$, Isil Ozer ${ }^{2}$, Mubeccel Demirkol$^{2}$, Tetsu Ebara ${ }^{1}$, Toshio Murase ${ }^{1}$, Teodor Podskarbi ${ }^{3}$, \\ Yoon S Shin ${ }^{3}$, Gulden Gokcay ${ }^{2}$ and Minoru Okubo ${ }^{1,4}$
}

Glycogen storage disease type III (GSD III) is an autosomal recessive disorder caused by deficiency in the glycogen debranching enzyme (gene symbol: $A G L$ ) with two enzyme activities: transferase and glucosidase. A missense mutation causing isolated glucosidase deficiency has never been reported. In this study, we examined 23 patients of Turkish ancestry and identified a novel missense mutation p.R1147G with isolated glucosidase deficiency, along with nine AGL mutations: six nonsense mutations (p.W373X, p.R595X, p.Q667X, p.Q1205X, p.W1327X and p.Q1376X), one deletion (c.1019delA) and two splicing mutation (c.293+2T $>$ G and c.958+1G $>$ A). As p.R1147G impaired glucosidase activity, but maintained transferase activity in vitro, a 12-year-old girl homozygous for p.R1147G was diagnosed with having isolated glucosidase deficiency. Of nine other mutations, p.W1327X and c.1019delA were recurrent, whereas seven mutations were novel. Six patients with p.W1327X were all from two nearby cities on the East Black Sea and shared the same AGL haplotype, indicating a founder effect in Turkish patients. Patients with the same mutations had identical haplotypes. Our results provide the first comprehensive overview of clinical and molecular features of Turkish GSD III patients and the first description of the missense mutation associated with isolated glucosidase deficiency.

Journal of Human Genetics (2009) 54, 681-686; doi:10.1038/jhg.2009.100; published online 16 October 2009

Keywords: AGL; glucosidase; glycogen storage disease type III; haplotype; mutation; transferase; Turkey

\section{INTRODUCTION}

Glycogen storage disease type III (GSD III; MIM \#232400) is an autosomal recessive inherited disorder characterized by fasting hypoglycemia, growth retardation, hepatomegaly, progressive myopathy and cardiomyopathy. ${ }^{1,2}$ GSD III is caused by a deficiency in the glycogen debranching enzyme, which has two independent catalytic activities: oligo-1, 4-1, 4-glucantransferase (EC 2.4.1.25) (transferase) and amylo-1, 6-glucosidase (EC 3.2.1.33) (glucosidase). Both activities are absent in the liver and the muscles in most patients with GSD III, which is named as subtype IIIa. Approximately $15 \%$ of the patients lose both two enzyme activity solely in the liver (subtype IIIb). Very few patients have been categorized to have selective loss of one of the two enzyme activities: isolated transferase deficiency (subtype IIId) and isolated glucosidase deficiency. Subtype IIIc was originally described as a reverse type of IIIb; one patient lost enzyme activity in muscle only. ${ }^{3,4}$ However, such patients have never been reported after the original patient, and the original meaning of IIIc apparently fell into oblivion. On the other hand, isolated glucosidase deficiency used to be labeled as other ${ }^{4}$ or IIIf, ${ }^{5}$ but at present, it has been named as IIIc. ${ }^{6}$ The molecular analysis of patients with isolated glucosidase deficiency has never reported. In this study, we present clinical and genetic features of a patient with isolated glucosidase deficiency. In vitro functional analysis of p.R1147G, which was identified in patient 1 , has been reported elsewhere. ${ }^{7}$

A gene coding human glycogen debranching enzyme (gene symbol: $A G L$ ) has been shown to be $85 \mathrm{~kb}$ in length and composed of 35 exons, encoding a 7.0-kb mRNA. ${ }^{8}$ Molecular analyses of GSD IIIa and IIIb have been performed in several ethnic populations and over 60 different AGL mutations have been reported to date (Human Gene Mutation Database; http://www.hgmd.cf.ac.uk/ac/index.php). Whereas only two cases have been reported in IIId, ${ }^{9,10}$ none have been reported in isolated glucosidase deficiency.

Depending on ethnic groups, the spectrum of $A G L$ mutations in GSD III varies. In ethnic groups with high rate of consanguinity, prevalent mutations have been reported. For example, specific mutations are prevalent in North African Jewish population and in an

${ }^{1}$ Okinaka Memorial Institute for Medical Research, Tokyo, Japan; ${ }^{2}$ Department of Pediatric Nutrition and Metabolism, Istanbul Medical Faculty, Istanbul University, Istanbul, Turkey; ${ }^{3}$ Molecular Genetics and Metabolism Laboratory, Munich, Germany and ${ }^{4}$ Department of Endocrinology and Metabolism, Toranomon Hospital, Tokyo, Japan Correspondence: Dr M Okubo, Medicine, Okinaka Memorial Institute for Medical Research and Toranomon Hospital, 2-2-2 Toranomon, Minato-ku, Tokyo 105-8470, Japan. E-mail: QFG00550@nifty.com

Received 12 July 2009; revised 6 September 2009; accepted 23 September 2009; published online 16 October 2009 


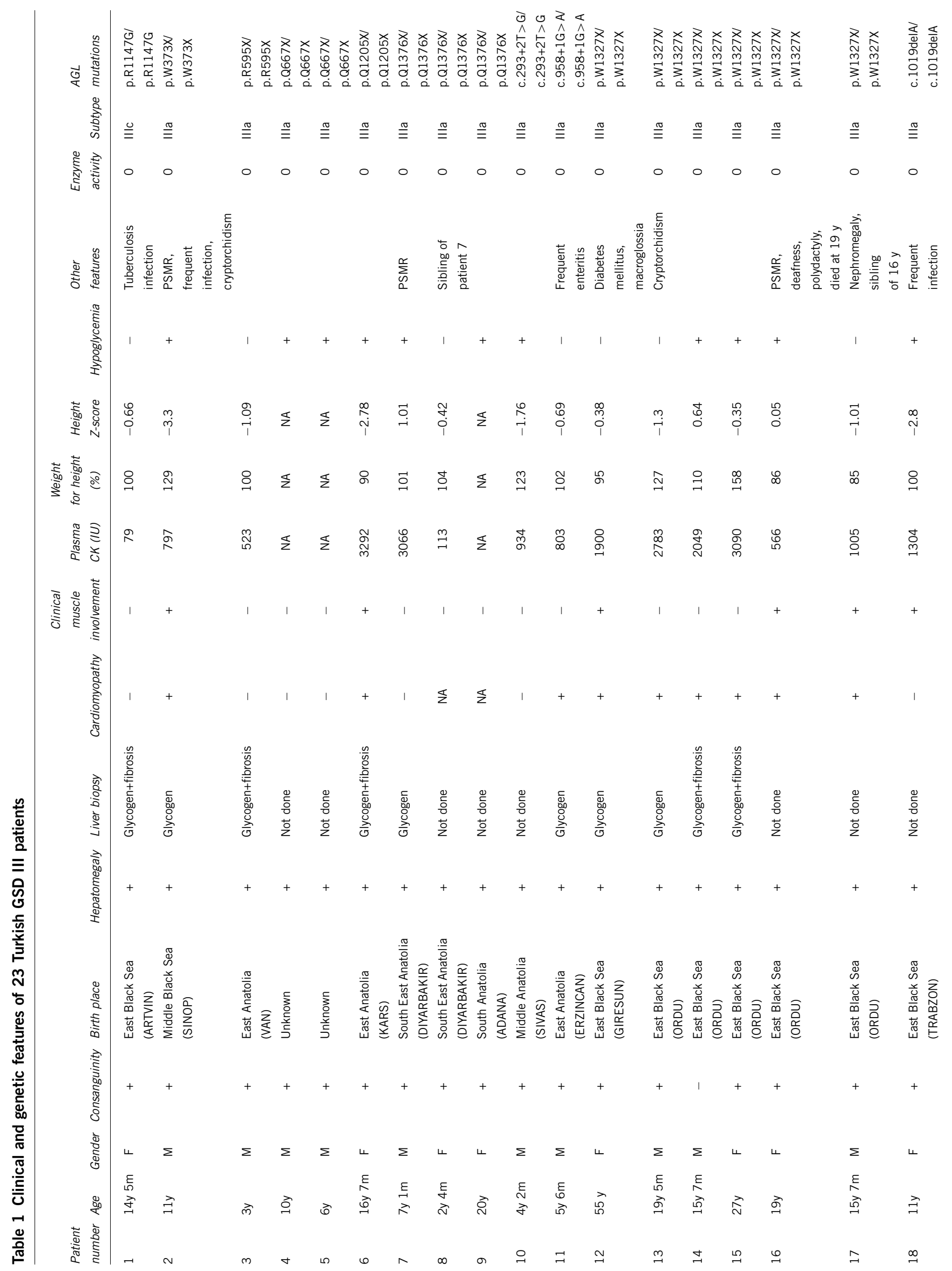




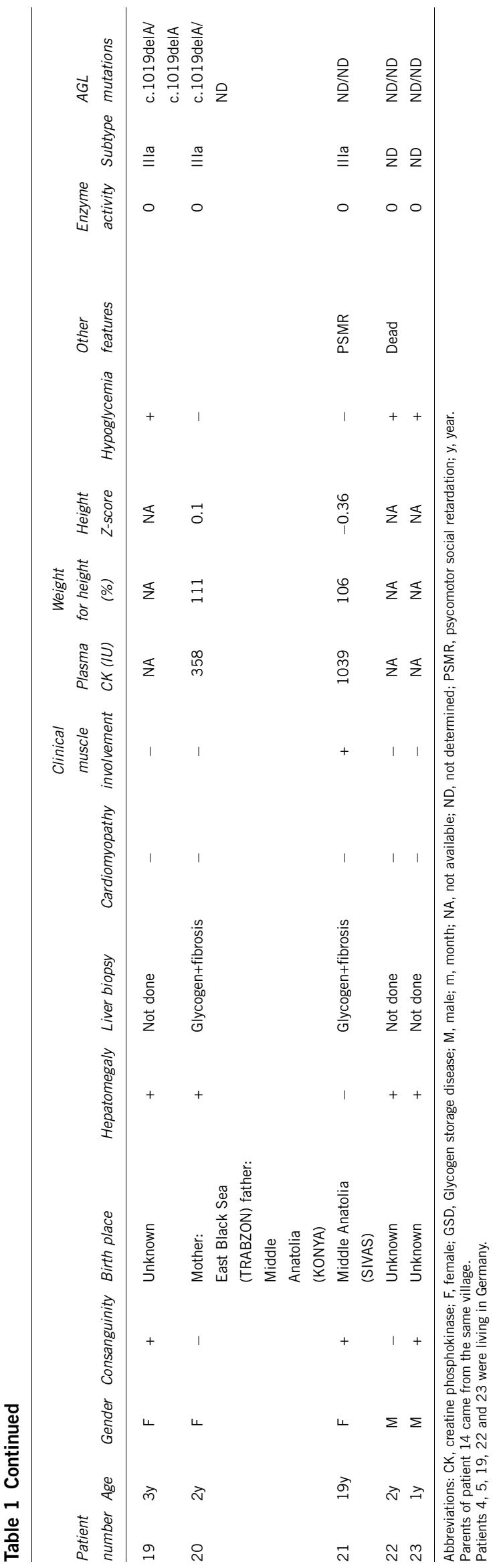

isolate such as Faroe Islands. ${ }^{11,12}$ On the contrary, genetic heterogeneity has been shown in other ethnic groups, such as Japanese, ${ }^{13}$ Italian $^{14}$ and Caucasian. ${ }^{15}$ In Turkey, the incidence of consanguineous marriages is generally high. However, we reported three different AGL mutations in three patients in Turkey: two splicing mutations c.846+5G $>A$ (IVS7+5G $>$ A) and c.2681+3dup (IVS21+5insA), ${ }^{16}$ along with one deletion (c.2474delC). ${ }^{17}$ To investigate further molecular characteristic in Turkey, we examined 23 new cases with Turkish GSD III.

\section{MATERIALS AND METHODS}

\section{Patients}

A total of 23 Turkish GSD III patients from 21 unrelated families were investigated. Patients 7 and 8 were siblings. Patients 16 and 17 were siblings as well. Clinical features were shown in Table 1. A total of 18 patients lived in Turkey and 5 in Germany. The patients were confirmed as having deficient debranching enzyme activity in peripheral blood cells by the method of Shin. ${ }^{18}$ Consanguinity was ascertained in 18 out of 21 families. The methods to evaluate cardiomyopathy were electrocardiography and echocardiography. The study was approved by local ethics committees and performed with the patients' and their families' informed consent.

Patient 1 is one of three children in a consanguineous family. Her parents were first cousins. Her sister died at birth and her brother died at age of 7 months due to cardiac insufficiency. She was first noticed to have hepatomegaly at age of 1 year and 3 months. Liver biopsy was performed at age 2 years and histological examinations showed glycogen accumulation and fibrosis. She suffered from tuberculosis when she was 9 years and 6 months old, and was treated for 6 months. Now patient 1 is 14 years and 5 months old, her physical and mental development have been normal. Her present clinical symptom is mild hepatomegaly only. She has experienced no episodes of hypoglycemia and has not showed muscle weakness on physical examination. Her electrocardiogram and echocardiogram are normal. Her plasma creatine phosphokinase level is $79 \mathrm{Ul}^{-1}$ (normal range: 62-287). Aspartate aminotransferase is $58 \mathrm{U} \mathrm{l}^{-1}$ (normal range: 5-32), alanine aminotransferase $84 \mathrm{U} \mathrm{l}^{-1}$ (normal range: 7-35), uric acid $3.7 \mathrm{mg}$ per $100 \mathrm{ml}$ (normal range: 2.6-6.0), triglycerides $218 \mathrm{mg}$ per $100 \mathrm{ml}$ (normal range $<150$ ), cholesterol $260 \mathrm{mg}$ per $100 \mathrm{ml}$ (normal range: $<220$ ) and lactate $0.8 \mathrm{mmoll}^{-1}$ (normal range $<2$ ).

\section{DNA sequence analysis of AGL}

Genomic DNA was isolated from peripheral blood leukocytes. The full coding exons, their relevant exon-intron boundaries and the $5^{\prime}$ - and $3^{\prime}$-flanking regions of the patients' $A G L$ were sequenced directly, as described previously. ${ }^{13}$ The nucleotides of $A G L$ cDNA were numbered according to $A G L$ isoform 1 (GenBank accession no. NM_000642).

Point mutations identified in patients were verified using restriction fragment length polymorphism (RFLP). RFLP analyses to detect p.W1327X and c.1019delA were described previously. ${ }^{16,19}$ Besides, a pair of primers (Table 2) were used for PCR and each specific restriction endonuclease was added to digest PCR products. Restriction digests were analyzed on a polyacrylamide gel. A total of 50 Turkish control subjects were examined by RFLP in the same manner to rule out the possibility that they are mere polymorphisms in controls.

\section{Haplotype determination in AGL}

A total of 25 single nucleotide polymorphisms (SNPs) in AGL were determined, as described previously. ${ }^{16}$ SNPs numbers were cited according to database of Single Nucleotide Polymorphisms (dbSNP Build 130) available from http:// www.ncbi.nlm.nih.gov/SNP/.

\section{Bioinformatical analysis}

Alignment of amino acid sequences from various species was performed using ClustalW2 (http://www.ebi.ac.uk/Tools/clustalw2/). ${ }^{20}$

\section{RESULTS}

We identified 37 mutant alleles (Table 1) and found 10 different AGL mutations (Table 3 ) in Turkish patients. Eight mutations were novel 
Table 2 RFLP detection for AGL mutations

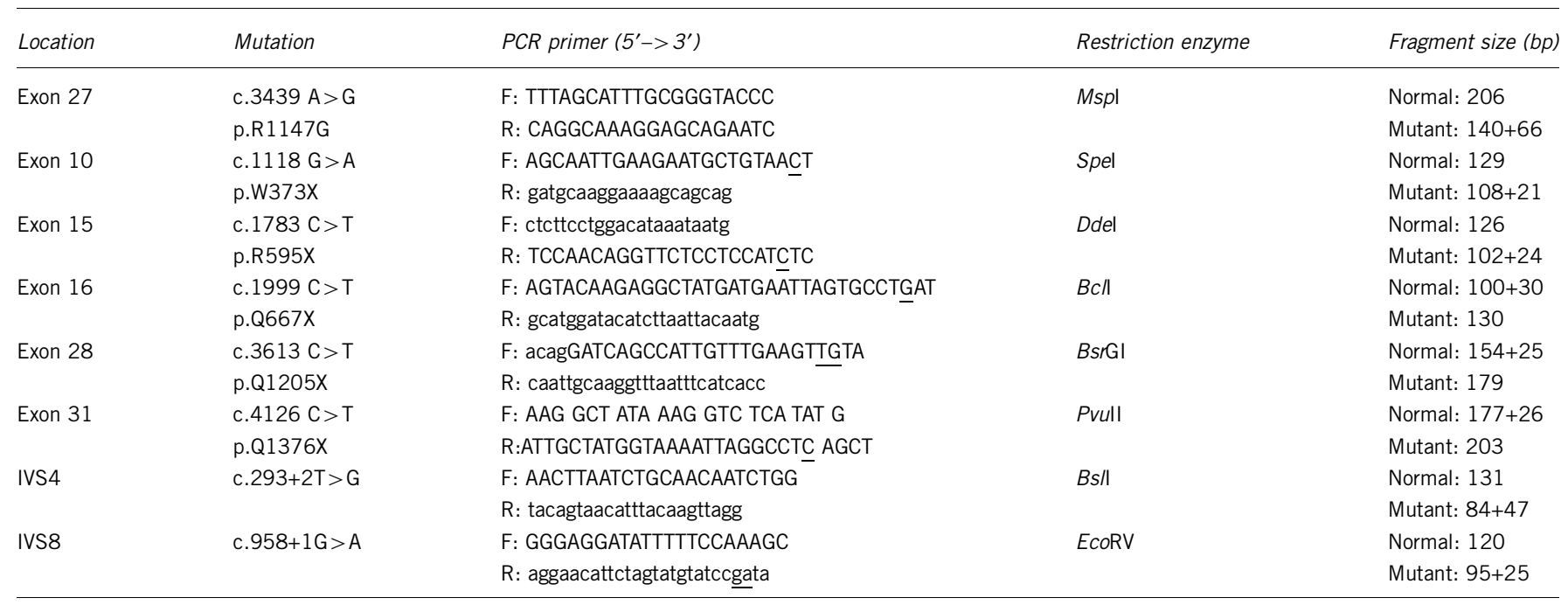

Abbreviations: F, forward; R, reverse; RFLP, restriction fragment length polymorphism.

Exon sequences are represented by uppercase letters and introns by lowercase letters.

Mismatch nucleotides are underlined.

Table 3 AGL mutations and haplotypes constructed by 25 SNPs

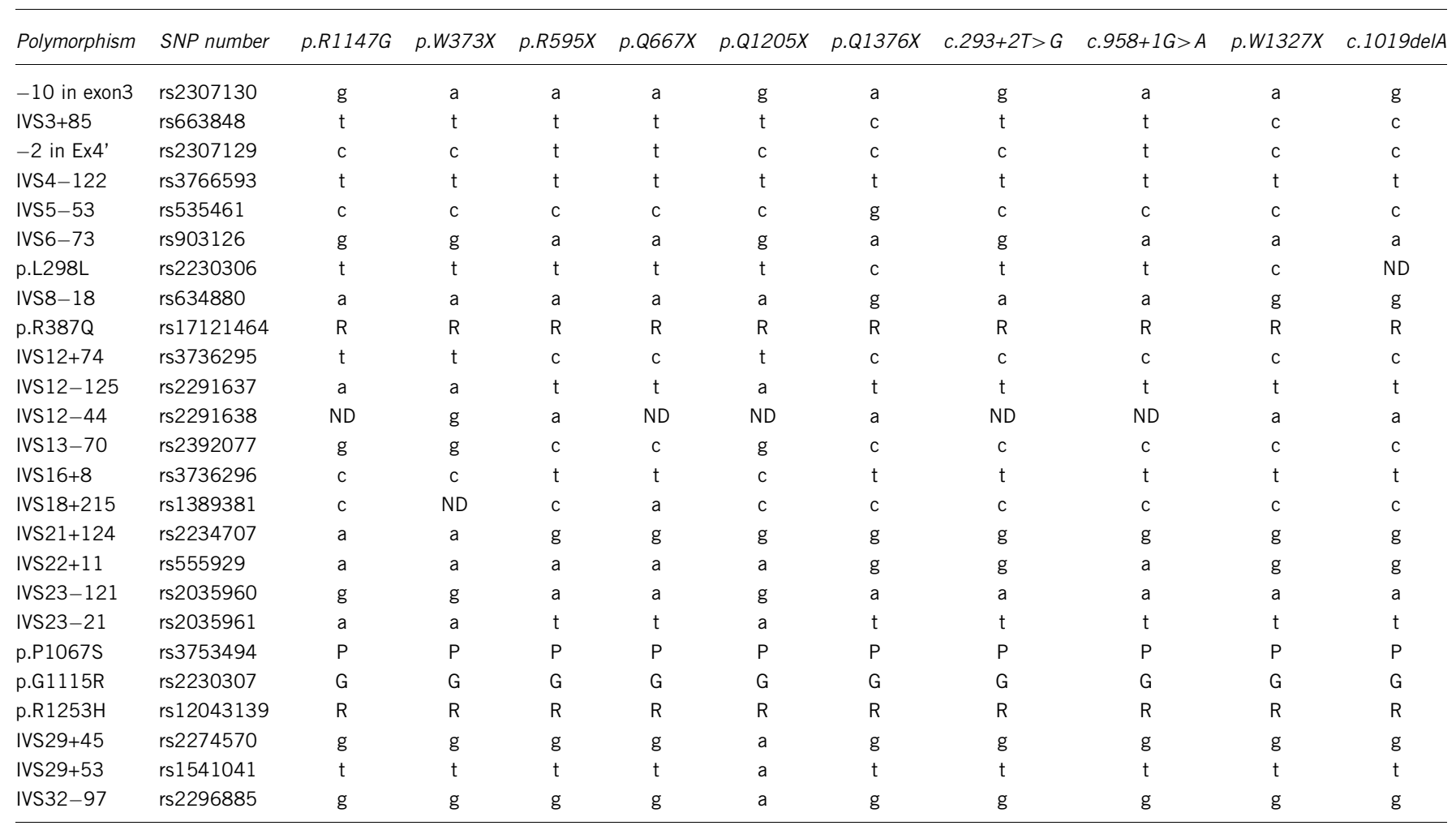

Abbreviations: ND, not determined; SNP, single nucleotide polymorphisms.

and two recurrent. All 10 mutations were verified by RFLP analyses and were not found in 50 normal Turkish controls (100 chromosomes).

Patient 1 was homozygous for a missense mutation in exon 27. Sequencing analysis showed an A-to-G substitution at nucleotide 3439, which replaces arginine by glycine at codon 1147 (p.R1147G).
RFLP analysis with MspI showed that patient 1 was homozygous for p.R1147G. No mutations except p.R1147G have been found in patient 1. Moreover, comparison of amino acid sequences among various species showed that arginine at codon 1147 was well preserved during evolution (Figure 1). 


Human
Chimpanzee
Cow
Horse
Rabbit
Dog
Mouse
Rat
Chicken
Fruit fly
Mosquito
Zebra fish
C.elegans
Yeast

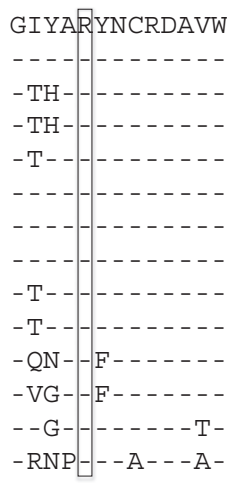

Figure 1 Comparison of amino acid sequences around human 1147 amino acid of glycogen debranching enzymes from various species. Identical amino acids are represented as -. The human arginine at codon 1147 and corresponding amino acids are enclosed within a box. The species and the NCBI accession numbers are as follows: Human (Homo sapiens), NP_000019; Chimpanzee (Pan troglodytes), XP_524777; Cow (Bos taurus) predicted protein, XP_595566; Horse (Equus caballus), NP_001103778; Rabbit (Oryctolagus cuniculus), NP_001075716; Dog (Canis familiaris), XP_537057; Mouse (Mus musculus), NP_001074795; Rat (Rattus norvegicus), NP_001102034; Chicken (Gallus gallus) predicted protein, XP_422317; Fruitfly (Drosophila melanogaster), NP_726062; Mosquito (Anopheles gambiae), XP_321957; Zebra fish (Danio rerio) predicted protein, XP_696194; Caenorhabditis elegans hypothetical protein, NP_496984; Yeast (Saccharomyces cerevisiae), NP_015510.

Sequence analysis of patient 2 showed a G-to-A substitution at nucleotide 1118 in exon 10, resulting in premature termination at codon 373 (p.W373X). Patient 3 was a homozygote for p.R595X. In sequencing, a C-to-T substitution at nucleotide 1783 was found, which changes arginine to stop codon at codon 595 (p.R595X). Sequence analysis of patients 4 and 5 showed a C-to-T substitution at nucleotide 1999 in exon 16, causing premature termination at codon 667 (p.Q667X). Patient 6 had a C-to-T substitution at nucleotide 3613 in exon 28, replacing glutamine by a stop codon (p.Q1205X). Patient 7 was shown to be homozygous for a nonsense mutation. Sequencing analysis showed a C-to-T transition at nucleotide 4126 in exon 31, resulting in substitution of glutamine at codon 1376 by termination codon (p.Q1376X). RFLP analysis with PvuII indicated that patients 7,8 and 9 were homozygotes for p.Q1376X. Patient 10 was homozygous for a T-to-G substitution at the second nucleotide of intron 4 (c.293+2T $>\mathrm{G})$. Sequence analysis of patient 11 indicated a G-to-A substitution at the first nucleotide of intron 8 (c. $958+1 \mathrm{G}>\mathrm{A})$.

Sequence analysis of patient 12 revealed a G-to-A substitution at nucleotide 3980 in exon 31 that replaces tryptophan by termination codon at codon 1327 (p.W1327X). RFLP analysis with XbaI, as described previously, ${ }^{19}$ indicated that six patients $12,13,14,15,16$ and 17 were homozygous for p.W1327X.

In patient 18 , sequencing analysis revealed deletion of $\mathrm{A}$ at nucleotide 1019 in exon 9 (c.1019delA), leading to a premature termination because of frameshift. RFLP analysis with EcoRI, as described previously, ${ }^{16}$ showed that patients 18 and 19 were homozygotes and patient 20 was heterozygote for c.1019delA. Sequencing analysis did not detect the other mutation of patient 20 .

No mutations were detected in patients 21, 22 and 23. Patients had neither c.846+5G > A, c.2681+3dup nor c.2474delC, each of which was reported in Turkish GSD III. In addition, mutations in exon 3, reportedly associated with GSD IIIb, were not detected in any of 23 patients.

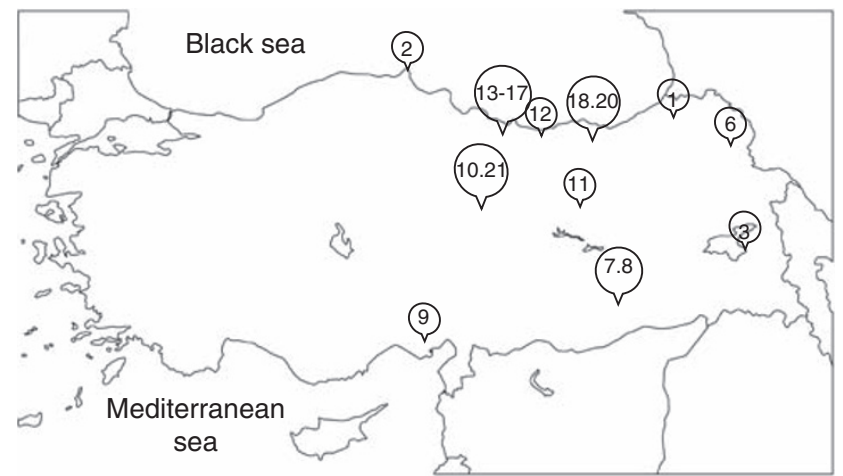

Figure 2 Geographic distribution of glycogen storage disease type III (GSD III) patients in Turkey. Figures in circles indicate patient number. Patients 4, 5, 19, 22 and 23 are not shown, because they live in Germany.

Geographic distribution of patients in Turkey was shown in Figure 2. Six patients with p.W1327X were all form two nearby cities on the East Black Sea.

AGL haplotype analysis of 10 mutations was shown in Table 3. Six patients with p.W1327X shared the same haplotype. Patients with same mutations, that is, two with p.Q667X, three with p.Q1376X and three with c.1019delA had identical haplotypes. These results indicated that patients with same mutations were descendants from a common ancestor in Turkey. Moreover, each haplotype had at least one different SNP, although haplotypes with p.R595X and c.958+1G > A were identical except rs2291638, which we could not determine in patient 11 with c. $958+1 \mathrm{G}>\mathrm{A}$.

\section{DISCUSSION}

We have shown the allelic heterogeneity of AGL mutations in patients of Turkish ancestry. Our genetic analysis of 23 Turkish patients revealed 10 different mutations. Five novel nonsense mutations (p.W373X, p.R595X, p.Q667X, p.Q1205X and p.Q1376X,) are predicted to lead to premature termination, which completely abolishes the enzyme activity. Those premature stop codons are likely to be recognized by nonsense-mediated mRNA decay machinery, leading to absence of $A G L$ mRNA. Two splicing mutations (c.293+2T $>\mathrm{G}$ and c. $958+1 \mathrm{G}>\mathrm{A}$ ) are predicted to impair normal splicing, because the 5 -dinucleotides in the donor splice site should be GT for normal splicing. At the second nucleotide of the donor splice site in intron 4, the substitution by a different nucleotide $(c .293+2 \mathrm{~T}>\mathrm{A})$ was reported to cause aberrant splicing. ${ }^{21}$ Computational splice site analysis indicated that both mutations completely abolished the function of the donor splice site, suggesting that the mutation is likely to obliterate the splicing. ${ }^{22}$

A novel AGL missense mutation p.R1147G was identified in patient 1 . As far as arginine at codon 1147 is concerned, this amino acid is conserved across species and located in the C-terminal half of AGL. In addition, site-directed mutagenesis study in yeast glycogen debranching enzyme showed that amino acid substitutions in the C-terminal half lost glucosidase activity, but retained transferase activity. ${ }^{23}$ Moreover, we expressed p.R1147G mutant and normal AGL proteins in COS cells transiently, and measured both glucosidase and transferase activities. ${ }^{7}$ The p.R1147G exhibited negligible glucosidase activity, but retained $40 \%$ of transferase activity compared with wild AGL. p.R1147G might alter the tertiary conformation of the enzyme and slightly reduce the transferase activity. However, transferase activity level is not deficient and this is different from IIIa. 
Interestingly, p.R1147G displayed increase ubiquitination, though protein stability was not altered. We conclude that the diagnosis of patient 1 is isolated glucosidase deficiency. In the literature, there have been only one twin case with isolated glucosidase deficiency. ${ }^{4}$ To our knowledge, p.R1147G is the first missense mutation associated with isolated glucosidase deficiency. Clinically, patient 1 had mild hepatomegaly, but showed neither clinical muscle involvement nor hypoglycemia. Follow-up of patient 1 is under way.

p.W1327X is a prevalent mutation in Turkish GSD III patients. All six patients come from two nearby cities on the East Black Sea. $A G L$ haplotype analysis showed that all six patients had the same haplotype, indicating a founder effect, that is, they had a common ancestor. As p.W1327X was reported in a Tunisian, ${ }^{24}$ an Egyptian, ${ }^{19}$ a Caucasian from Canada ${ }^{16}$ and a German-Ukraine ${ }^{25}$ patient, we compared AGL haplotypes of Egyptian, Caucasian and Turkish patients studied by us to determine whether the mutation occurred independently. Three haplotypes were different: (1) the Egyptian had $t$ in rs2307129, whereas Turkish and Caucasian had c; (2) the Caucasian had a in rs1541041, whereas Turkish and Egyptian had t. These results suggested that $\mathrm{W} 1327 \mathrm{X}$ is a recurrent mutation in various ethnic groups.

As for clinical features, we could not observe robust phenotypegenotype relationship. Even in patients with the same mutation, clinical features varied. For example, patients with p.W1327X had unique clinical courses. Patient 12 had macroglossia inhibiting her ability to speak at diagnosis. After the onset of dietary treatment with high protein, the symptom dissipated. As she was the eldest patient in the group, this problem may be associated with late diagnosis at an older age. Patient 16 had severe muscle weakness and died from cardiac failure at the age of 19 years, whereas patient 12 lives to the age of 55 and with mild muscle involvement. Other factors modulating the progression of clinical features may exist and further studies will be needed to elucidate them.

In summary, we showed the first comprehensive overview of clinical and molecular features of Turkish GSD III patients. This is the first report of the missense mutation being associated with isolated glucosidase deficiency.

\section{CONFLICT OF INTEREST}

The authors declare no conflict of interest.

\section{ACKNOWLEDGEMENTS}

This study was supported in part by a research grant from the Nakatomi Foundation. We thank Yuichi Kondo for his technical assistance.

1 Chen, Y. T. in The Metabolic and Molecular Bases of Inherited Disease, 8th edn. (eds. Scriver, C.R., Beaudet, A.L., Sly, W.S. \& Valle, D.) 1521-1551 (McGraw-Hill, New York, 2001).

2 Shin, Y. S. Glycogen storage disease: clinical, biochemical, and molecular heterogeneity. Semin. Pediatr. Neurol. 13, 115-120 (2006).
3 Hers, H. G., Verhue, W. \& Mathieu, M. in Control of Glycogen Metabolism (eds. Whelan, W.J. \& Cameron, M.P.) 151-175 (J. \& A. Churchill, London, 1964).

4 Van Hoof, F. \& Hers, H. G. The subgroups of type 3 glycogenosis. Eur. J. Biochem. 2, 265-270 (1967).

5 Howell, R. R. \& Williams, J. C. in The Metabolic Basis of Inherited Disease, 5th edn. (eds. Stanbury, J.B., Wyngaarden, J.B., Fredrickson, D.S., Goldstein, J.L. \& Brown, M.S.) 141-166 (McGraw-Hill, New York, 1983).

6 Shen, J., Bao, Y., Liu, H. M., Lee, P., Leonard, J. V. \& Chen, Y. T. Mutations in exon 3 of the glycogen debranching enzyme gene are associated with glycogen storage disease type III that is differentially expressed in liver and muscle. J. Clin. Invest. 98, 352-357 (1996).

7 Cheng, A., Zhang, M., Okubo, M., Omichi, K. \& Saltiel, A. R. Distinct mutations in the glycogen debranching enzyme found in glycogen storage disease type III lead to impairment in diverse cellular functions. Hum. Mol. Genet. 18, 2045-2052 (2009).

8 Bao, Y., Dawson, T. L. Jr. \& Chen, Y. T. Human glycogen debranching enzyme gene (AGL): complete structural organization and characterization of the $5^{\prime}$ flanking region. Genomics 38, 155-165 (1996).

9 Sugie, H., Fukuda, T., Ito, M., Sugie, Y., Kojoh, T. \& Nonaka, I. Novel exon 11 skipping mutation in a patient with glycogen storage disease type IIId. J. Inherit. Metab. Dis. 24, 535-545 (2001).

10 Endo, Y., Fateen, E., El Shabrawy, M., Aoyama, Y., Ebara, T., Murase, T. et al. Egyptian glycogen storage disease type III-Identification of six novel AGL mutations, including a large $1.5 \mathrm{~kb}$ deletion and a missense mutation p.L620P with subtype IIId. Clin. Chem. Lab. Med. 47, 1233-1238 (2009).

11 Santer, R., Kinner, M., Steuerwald, U., Kjaergaard, S., Skovby, F., Simonsen, H. et al. Molecular genetic basis and prevalence of glycogen storage disease type IIIA in the Faroe Islands. Eur. J. Hum. Genet. 9, 388-391 (2001).

12 Parvari, R., Moses, S., Shen, J., Hershkovitz, E., Lerner, A. \& Chen, Y. T. A single-base deletion in the $3^{\prime}$-coding region of glycogen-debranching enzyme is prevalent in glycogen storage disease type IIIA in a population of North African Jewish patients. Eur. J. Hum. Genet. 5, 266-270 (1997).

13 Okubo, M., Horinishi, A., Takeuchi, M., Suzuki, Y., Sakura, N., Hasegawa, Y. et al. Heterogeneous mutations in the glycogen-debranching enzyme gene are responsible for glycogen storage disease type IIla in Japan. Hum. Genet. 106, 108-115 (2000).

14 Lucchiari, S., Pagliarani, S., Salani, S., Filocamo, M., Di Rocco, M., Melis, D. et al. Hepatic and neuromuscular forms of glycogenosis type III: nine mutations in AGL. Hum. Mutat. 27, 600-601 (2006).

15 Shaiu, W. L., Kishnani, P. S., Shen, J., Liu, H. M. \& Chen, Y. T. Genotype-phenotype correlation in two frequent mutations and mutation update in type III glycogen storage disease. Mol. Genet. Metab. 69, 16-23 (2000).

16 Endo, Y., Horinishi, A., Vorgerd, M., Aoyama, Y., Ebara, T., Murase, T. et al. Molecular analysis of the AGL gene: heterogeneity of mutations in patients with glycogen storage disease type III from Germany, Canada, Afghanistan, Iran, and Turkey. J. Hum. Genet. 51, 958-963 (2006).

17 Aoyama, Y., Endo, Y., Ebara, T., Murase, T., Shin, Y. S., Podskarbi, T. et al. A novel AGL mutation in a Turkish patient with glycogen storage disease type IIla. Pediatr. Int. (in press).

18 Shin, Y. S., Ungar, R., Rieth, M. \& Endres, W. A simple assay for amylo-1,6-glucosidase to detect heterozygotes for glycogenosis type III in erythrocytes. Clin. Chem. 30, 1717-1718 (1984).

19 Endo, Y., Fateen, E., Aoyama, Y., Horinishi, A., Ebara, T., Murase, T. et al. Molecular characterization of Egyptian patients with glycogen storage disease type IIla. J. Hum. Genet. 50, 538-542 (2005).

20 Larkin, M. A., Blackshields, G., Brown, N. P., Chenna, R., McGettigan, P. A., McWilliam, $H$. et al. Clustal $W$ and Clustal $X$ version 2.0. Bioinformatics. 23, 2947-2948 (2007).

21 Hadjigeorgiou, G. M., Comi, G. P., Bordoni, A., Shen, J., Chen, Y. T., Salani, S. et al. Novel donor splice site mutations of AGL gene in glycogen storage disease type Illa. J. Inherit. Metab. Dis. 22, 762-763 (1999).

22 Nalla, V. K. \& Rogan, P. K. Automated splicing mutation analysis by information theory. Hum. Mutat. 25, 334-342 (2005). Erratum in Hum. Mutat. 29, 1168 (2008).

23 Nakayama, A., Yamamoto, K. \& Tabata, S. Identification of the catalytic residues of bifunctional glycogen debranching enzyme. J. Biol. Chem. 276, 28824-28828 (2001).

24 Lucchiari, S., Donati, M. A., Parini, R., Melis, D., Gatti, R., Bresolin, N. et al. Molecular characterisation of GSD III subjects and identification of six novel mutations in AGL. Hum. Mutat. 20, 480 (2002).

25 Schoser, B., Glaser, D. \& Muller-Hocker, J. Clinicopathological analysis of the homozygous p.W1327X AGL mutation in glycogen storage disease type 3. Am. J. Med. Genet. 146A, 2911-2915 (2008). 